\title{
Uma Nota Sobre "Teste da Convergência do PIB per capita da Agropecuária no Brasil"
}

\author{
Christiano Penna ${ }^{1}$ e Fabricio Linhares ${ }^{2}$
}

Resumo: A presente nota discute a contradição entre os testes de $\beta$ e $\sigma$-convergência dos PIBs agropecuários per capita recentemente apresentada por Spohr e Freitas (RESR, vol. 49, no 02, p. 341-368, abr/jun 2011). Enquanto o primeiro tipo de teste sustenta a hipótese de convergência, o teste da $\sigma$ - convergência não valida tal hipótese. Aqui se argumenta que esta contradição possivelmente advém de não linearidades no processo de convergência, que seriam condizentes com o processo de formação de clubes de convergência. O teste da $\beta$ - convergência é refeito de modo mais rigoroso, de acordo com a metodologia proposta em Caselli, Esquivel e Lefort (1996) e, posteriormente, a formação de clubes de convergência é testada através da metodologia proposta em Phillips e Sul (2007), que tem a vantagem de acomodar diversos tipos de heterogeneidade. A formação de três clubes de convergência é constatada e busca-se esclarecer a contradição anteriormente reportada.

Palavras-chaves: agropecuária, testes de convergência, PIB agropecuário per capita, modelos não lineares.

\begin{abstract}
This note discusses the contradiction between $\beta$ and $\sigma$-convergence tests of agricultural per capita GDP, which was recently presented by Spohr and Freitas (RESR, vol. 49, No. 02, p. 341-368, April / June 2011). While the first type of test supports the hypothesis of convergence, the $\sigma$-convergence test does not validate this hypothesis. It is argued that this contradiction probably arises from nonlinearities in the convergence process, which can characterize the formation of convergence clubs. The convergence test was done again in a more rigorous way, according to the methodology proposed by Caselli, Eesquivel and Lefort (1996) and, subsequently, the formation of convergence clubs is tested using the methodology proposed in Phillips and Sul (2007), which has the advantage of accommodating various types of heterogeneity. The formation of three convergence clubs is confirmed and these results try to clarify the contradiction previously reported.
\end{abstract}

Key-words: agriculture, convergence tests, agricultural per capita GDP, nonlinear models.

Classificação JEL: Q19, E13, C23.

1 Professor da UFC/Sobral. E-mail: cmp@caen.ufc.br

2 Professor do Caaen/UFC. E-mail: flinhares@caen.ufc.br 


\section{Introdução}

Sporh e Freitas (2011) apresentaram recentemente nesta revista (RESR, vol. 49, n02, p. 341-368, abr/jun 2011) testes da hipótese da convergência do PIB agropecuário per capita (PIBAGpc) em termos de $\sigma$ e $\beta$ - convergência absoluta e condicional. Estes dois conceitos têm seus alicerces na teoria neoclássica de crescimento e sugerem que: quando a dispersão da renda real per capita entre um grupo de economias se reduz ao longo do tempo, diz-se que ocorre $\sigma$ convergência e, quando a correlação parcial entre a taxa de crescimento da renda real per capita e o nível de renda real inicial é negativo, diz-se que há $\beta$ - convergência; se esta correlação negativa for não condicionada a características estruturais comuns entre as economias, diz-se que a $\beta$ convergência ocorre em termos absolutos; já se a correlação for condicionada a tais características, diz que há $\beta$ - convergência condicional. ${ }^{3}$

Os autores relatam que os testes da $\beta$ - convergência, tanto absoluta quanto condicional, suportam a hipótese de convergência. Entretanto, o teste com base na $\sigma$ - convergência conduzido por Spohr e Freitas (2011) sugere que a dispersão entre os PIBAGpc vem se ampliando com o tempo, o que contradiz a hipótese de convergência. ${ }^{4}$

3 Todas estas questões são muito bem expostas e discutidas em Spohr e Freitas (2011). Havendo necessidade, o leitor pode se remeter ao referido trabalho.

4 Isto está de acordo com a constatação da $\beta$ - convergência absoluta, em âmbito microrregional, feita em Almeida, Perobeli e Ferreira (2008), e com a constatação de $\beta$ convergência condicional, em âmbito estadual, feita por Barreto e Almeida (2009).
A controvérsia entre estes testes, além de ter implicações importantes para as políticas públicas, motiva novas pesquisas científicas e uma delas, como sugerem os autores, é a de que se adentre na questão de clubes de convergência. ${ }^{5}$

Esta nota tenta preencher algumas lacunas empíricas deixadas em aberto no trabalho de Spohr e Freitas (2011), além de dar continuidade à análise destes autores. Aanálise da $\sigma$-convergência é realizada aqui de modo um pouco mais simples, embora mais ilustrativo, e realizou-se o teste da $\beta$ - convergência com base no estimador GMM em primeiras diferenças; em ambos os casos, nossa análise corrobora com a análise de Sporh e Freitas (2011), o que agrega robustez aos resultados destes autores. Posteriormente, adentra-se na questão da formação de clubes de convergência. Isto é feito com base na metodologia de Phillips e Sul (2007), que é uma abordagem de séries temporais capaz de identificar clubes de convergência sobre o pressuposto de diversos tipos de heterogeneidade. Esta metodologia é capaz de absorver uma série de particularidades associadas à realidade da agropecuária brasileira, o que a torna bastante atrativa. Por fim, com base na evidência de clubes de convergência para os PIBAGpc estaduais, procura-se dirimir a controvérsia entre os testes de convergência inicialmente apresentados.

\footnotetext{
5 O processo de formação de clubes de convergência sugere que grupos de economias estariam convergindo para PIBAGpc de longo prazo distintos.
} 
Figura 1. Desvio médio dos PIBAGpc estaduais entre 1980 e 2008

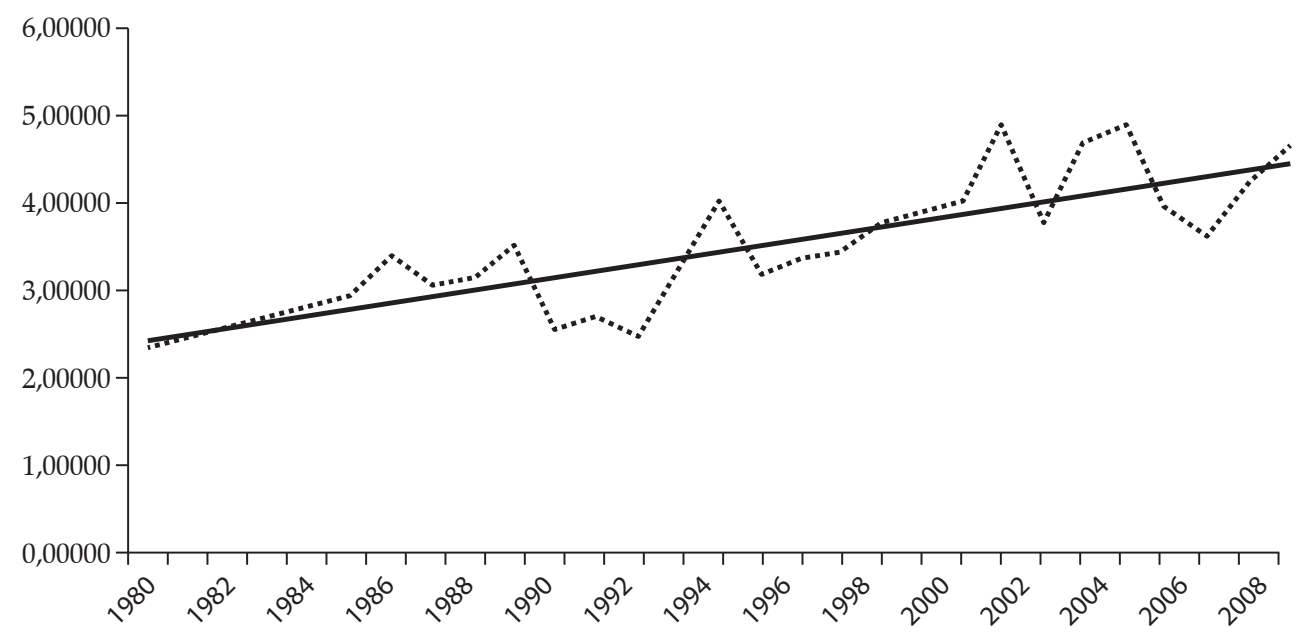

Fonte: Elaboração dos autores com base em dados do Ipeadata, descritos no Apêndice.

\section{Sigma convergência}

Um teste simples com base na hipótese da $\sigma$ convergência pode ser diretamente aplicado a um painel de dados; basta que se veja o comportamento de uma medida da variabilidade do conjunto de dados invariável à média. Uma boa métrica neste sentido é a média aritmética dos desvios médios dos pontos de dados a partir de sua média, ou seja, $(1 / n) \sum(x-\bar{x})$. Com base nos dados de PIBAGpc descritos no Apêndice, a Figura 1, sugere que esta medida apresenta uma tendência crescente ao longo do tempo, o que sugere, assim como notado por Spohr e Freitas (2011), que a hipótese da $\sigma$ convergência é violada. ${ }^{6}$

Posto que a $\sigma$ - convergência é violada, resta saber se há indícios de que a hipótese de $\beta$ - convergência não é transgredida, conforme sustentam Sporh e Freitas (2011).

\section{Beta Convergência}

Sporh e Freitas (2011) investigaram a hipótese de $\beta$-convergência por meio de uma análise de

6 O teste de $\sigma$ - convergência conduzido em Sporh e Freitas (2011) também foi refeito e apontou para os mesmos resultados. dados em painel que, basicamente, segue a seguinte estrutura: ${ }^{7}$

$$
\begin{aligned}
& (1 / \tau) \ln \left(\mathrm{y}_{\mathrm{i}, \mathrm{t}} / \mathrm{y}_{\mathrm{i}, \mathrm{t}-\tau}\right)= \\
& =\alpha_{\mathrm{i}}+\eta_{\mathrm{t}}+\rho 1 \ln \left(\mathrm{y}_{\mathrm{i}, \mathrm{t}-\tau}\right)+\mathrm{Z}_{\mathrm{i}, \mathrm{t}}^{\prime} \psi+\varepsilon_{\mathrm{i}, \mathrm{t}}
\end{aligned}
$$

em que a variável dependente é a taxa média de crescimento da renda per capita da economia i entre o período inicial, $\mathrm{y}_{\mathrm{i}, t-\tau}$ e final, $\mathrm{y}_{\mathrm{i}, \mathrm{t}} \alpha_{\mathrm{i}}$ é o efeito fixo capaz de controlar o componente idiossincrático; $\eta_{\mathrm{t}}$ é o efeito aleatório; o parâmetro $\rho$, a ser estimado, equivale a $-\mathrm{T}^{-1}\left(1-\mathrm{e}^{-\beta \mathrm{T}}\right)$, em que $\beta$ pode ser entendido como a velocidade de convergência para o estado estacionário; $\psi$ é um vetor de parâmetros a serem estimados e $\mathrm{Z}_{\mathrm{i}, \mathrm{t}}$ é um conjunto de variáveis adicionais de controle ${ }^{8}$, e $\varepsilon_{\mathrm{i}}$ representa o termo de erro.

A Figura 2 plota as taxas médias de crescimento contra o logaritmo neperiano dos PIBAGpcs estaduais levando-se em consideração os anos para os quais se tem informação relativamente fidedigna (1980, 1991, 1996, 2000

7 A equação (1) é uma formalização da equação apresentada em (38) no trabalho de Spohr e Freitas (2011).

8 Spohr e Freitas (2011), por exemplo, utilizaram os anos médios de escolaridade dos trabalhadores no setor agropecuário como variável de controle. 
Figura 2. Taxa média de crescimento contra PIBAGpc inicial

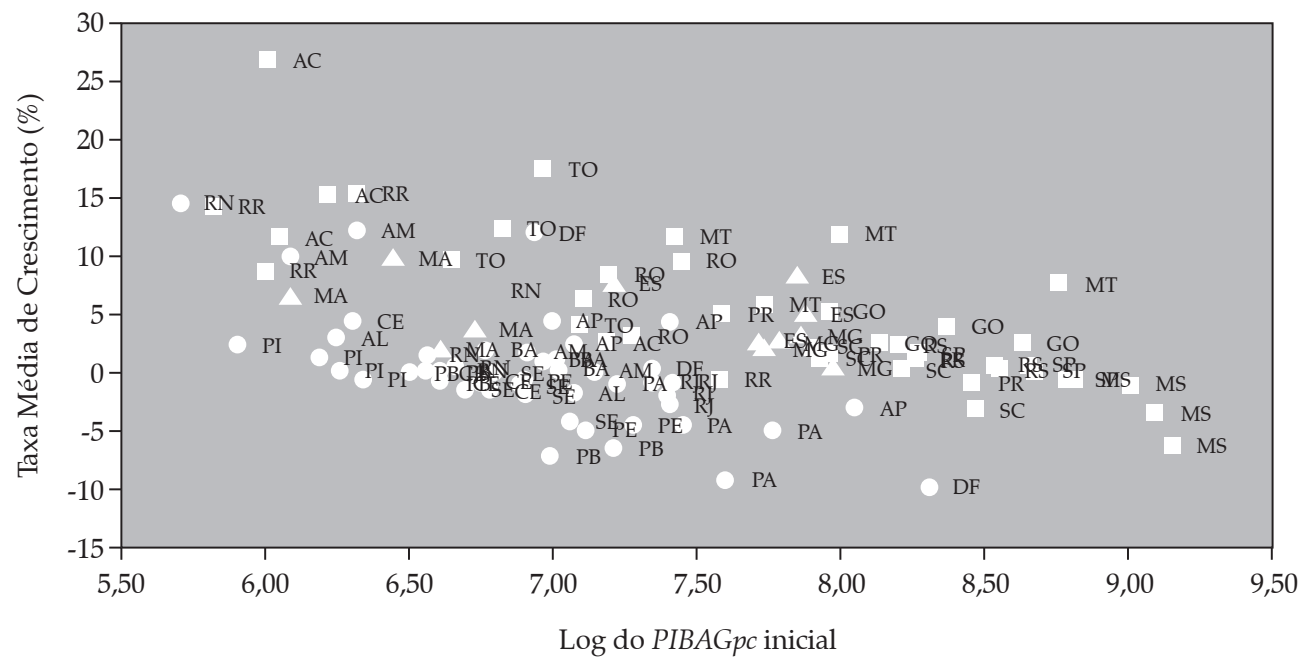

Fonte: Elaboração dos autores.

e 2007). ${ }^{9}$ Os pontos amostrais foram construídos conforme sugestão de Sporh e Freitas (2011), ou seja, tomando-se as taxas médias de crescimento dentre os demais anos e 2007. Foram destacados três grupos de estados, os quais serão discutidos em mais detalhes ao final do estudo. $\mathrm{O}$ que se observa da referida figura é padrão esperado associado à hipótese da $\beta$-convergência, ou seja, uma relação negativa entre taxa de crescimento e PIBAG per capita inicial, embora tal padrão pareça ser consistente com um modelo não linear.

Islam (1995), Caselli, Esquivel e Lefort (1996) e Bond, Hoeffler e Temple (2001) ressaltam o caráter endógeno do regressor $1 \mathrm{n}\left(\mathrm{y}_{\mathrm{i}, \mathrm{t}-\tau}\right)$ em (1) e sugerem que esta regressão se equipara a seguinte equação de painel dinâmico:

9 Para 2007 e 1996 a população rural foi tomada com base na contagem da população e, para os demais anos, com base no censo demográfico. Em relação ao PIB Agropecuário, para o ano de 1980 utilizou-se o Produto Interno Bruto a custo de fatores elaborado pelo IBGE; para 1991 e 1996 utilizou-se o Antigo Sistema de Contas Regionais e ara 2000 e 2007 utilizou-se o Sistema de Contas Regionais, Referência 2002 (As estimativas do PIB não são consistentes com o PIB por atividades. O problema se deve à utilização de conceitos distintos. O PIB refere-se a preços de mercado e as atividades a preços básicos). Diferente do feito por Spohr e Freitas (2011), o ano final aqui é o de 2007, em vez de 2004.

$$
\begin{aligned}
& \ln \left(\mathrm{y}_{\mathrm{i}, \mathrm{t}} / \mathrm{y}_{\mathrm{i}, \mathrm{t}-\tau}\right)=\alpha_{\mathrm{i}}+\eta_{\mathrm{t}}+\psi \ln \left(\mathrm{y}_{\mathrm{i}, \mathrm{t}-\tau} / \mathrm{y}_{\mathrm{i}, \mathrm{t}-2 \tau}\right)+ \\
& +\rho \ln \left(\mathrm{y}_{\mathrm{i}, \mathrm{t}-\tau}\right)+\varepsilon_{\mathrm{i}, \mathrm{t}}
\end{aligned}
$$

em que, $\rho=-\mathrm{T}^{-1}\left(1-\mathrm{e}^{-\beta \mathrm{T}}\right)<0$ e os demais parâmetros e variáveis têm interpretação e descriçãosemelhantesàsdiscutidasanteriormente. Note-se que a equação (2) representa um modelo de painel dinâmico cuja estrutura é um processo AR(1) na taxa de crescimento do PIBAGpc. Greene (2003) ressalta que modelos dinâmicos deste tipo são relativamente atraentes, pois a variável dependente defasada seria capaz de incorporar o efeito de uma série de outras variáveis explicativas no modelo, ou seja, é de se esperar que a taxa de crescimento defasada, $\ln \left(\mathrm{y}_{\mathrm{i}, \mathrm{t}-\tau} / \mathrm{y}_{\mathrm{i}, \mathrm{t}-2 \tau}\right)$ incorpore $\mathrm{o}$ efeito de todas as possíveis variáveis associadas à matriz de controle, $Z_{i, t}$ daí a associação do parâmetro $\psi$ à taxa de crescimento defasada nesta segunda especificação.

Com base nos dados descritos no apêndice, a equação (2) foi estimada levando-se em consideração $\tau=4$ (ou seja, os dados longitudinais consideram as taxas médias de crescimento entre 1980-1984, 1984-1992, 1992-1996, 1996-2000, 2000-2004 e 2004-2008, além dos PIBAGpc iniciais de cada um destes subperíodos). Os resultados são os que se seguem na Tabela 1: 
Tabela 1. Teste de beta-convergência para os PIBAGpc estaduais

\begin{tabular}{cccc}
\multicolumn{5}{c}{$\ln \left(\mathrm{y}_{\mathrm{i}, \mathrm{t}} / \mathrm{y}_{\mathrm{i}, \mathrm{t}-\tau}\right)=\alpha_{\mathrm{i}}+\eta_{\mathrm{t}}+\psi \ln \left(\mathrm{y}_{\mathrm{i}, \mathrm{t}-\tau} / \mathrm{y}_{\mathrm{i}, \mathrm{t}-\mathrm{-} \tau}\right)+\rho \ln \left(\mathrm{y}_{\mathrm{i}, \mathrm{t}-\tau}\right)+\varepsilon_{\mathrm{i}, \mathrm{t}}$} \\
\hline Parâmetro & Coeficiente & Estatística t & P-valor \\
\hline$\psi$ & -0.432605 & -6.332933 & 0.0000 \\
$\rho$ & -0.046779 & -3.802124 & 0.0002 \\
\hline
\end{tabular}

Nota: Estimativas via GMM First Difference com Efeitos Fixos.

Fonte: Elaboração dos autores.

O modelo estimado acima só é passível de análise caso a hipótese nula do teste de Sargan, a qual sugere que as variáveis instrumentais utilizadas são não correlacionadas com os resíduos, não seja rejeitada. A estatística J para o modelo é de 11.73280 , e o posto dos instrumentos é igual a 15. Isto retorna um p-valor para a estatística de Sargan de 0,5496, concluindo-se, portanto, que os instrumentos aqui utilizados são válidos.

Como prevê a teoria, observa-se que o coeficiente estimado associado à renda inicial é negativo e estatisticamente significativo; entretanto, tal coeficiente é menor do que o encontrado anteriormente: Spohr e Freitas (2011) encontram $\hat{\rho}=-0,070128$, enquanto aqui se tem $\hat{\rho}=-0,046779$. Estadiferença sedá, possivelmente, devido ao "viés da endogeneidade" ${ }^{10}$

Contudo, a diferença entre os estudos pouco difere em termos de análise do processo de convergência, isto porque as diferentes dimensões temporais utilizadas ${ }^{11}$ levam a velocidades de convergência muito próximas: $12,18 \%$ aqui e $13,68 \%$ naquele estudo; o que sugere uma meia-vida, em ambos os casos, de algo próximo de seis anos.

\section{Clubes de convergência: a abordagem de Phillips e Sul (2007)}

Em resumo, a análise até aqui delineada agrega robustez à análise de Spohr e Freitas (2011)

10 Uma discussão minuciosa dos possíveis tipos de viés associados à equação (1), assim como um exame mais detalhado das técnicas de estimação empregadas neste artigo podem ser vistas em Penna e Linhares $(2009,2011)$.

11 Aqui (1980-2008) e naquele estudo (1980-2004). e chega aos mesmos resultados encontrados anteriormente: embora haja sinais visíveis de violação da $\sigma$ - convergência, há fortes indícios em favor da ocorrência de $\beta$ - convergência.

Uma maneira de se buscar conciliar os testes de $\beta$ e $\sigma$-convergência é recorrer a análises mais sofisticadas, que adentrem, inclusive, na questão da formação de clubes de convergência. O estudo de Oliveira Junior, Castelar e Ferreira (2009) faz isso para microrregiões com base num modelo threshold, mas, talvez devido à escassez dos dados, não há na literatura análises do tipo no âmbito estadual. ${ }^{12}$

Se o intuito é identificar clubes de convergência sob arcabouço da $\beta$-convergência, seria condizente, então, utilizar um modelo de painel dinâmico com efeito threshold. Embora esta técnica aparentemente acomode bem a escassez de dados longitudinais para o caso da agropecuária brasileira, as ciências estatística e econométrica ainda não avançaram o suficiente para que tal metodologia seja empregada, mas, conforme a mesma avance, esta promete ser uma interessante linha de pesquisa. ${ }^{13}$

Esta seção apresenta uma primeira tentativa de se abordar o processo de formação de clubes de convergência dos PIBAGPc em âmbito estadual. Embora a qualidade dos dados não seja tão boa quanto se almeje, é importante ressaltar que a técnica aqui empregada não se concentra necessariamente nos dados reais dos PIBAGpc

\footnotetext{
12 Silva e Oliveira Junior (2010) também fazem uma análise com base em técnicas de Econometria Espacial para os municípios da Amazônia Legal.

13 Shin (2008) já prospecta alguns avanços neste sentido, entretanto, seu trabalho ainda não foi concluído.
} 
estaduais, mas, sim, nas tendências de longo prazo dos mesmos.

Mais especificamente, embora a metodologia proposta em Phillips e Sul (2007) necessite de um painel que leve em conta um período relativamente longo, a mesma faz uso dos PIBAGpc dos estados sem o componente de ciclos econômicos, ou seja, requer as séries filtradas dos PIBAGpc. ${ }^{14}$ Com efeito, ao contrário de grande parte das análises de séries temporais, neste caso, a indisponibilidade de dados não parece ser um impeditivo para o exercício.

A ideia básica do teste de Phillips e Sul (2007) se concentra na seguinte regressão semiparamétrica, também chamada de teste log $t:{ }^{15}$

$$
\begin{aligned}
& \log \frac{\mathrm{H}_{1}}{\mathrm{H}_{\mathrm{t}}}-2 \log [\log t]=\beta_{0}+\beta_{1} \log t+\mathrm{u}_{\mathrm{t}} \\
& \text { para } \\
& t=\mathrm{T}_{0}, \ldots, \mathrm{T}
\end{aligned}
$$

em que $\mathrm{H}_{1} / \mathrm{H}_{\mathrm{t}}$ representa a relação de variância cross-section encontrada por meio de $\mathrm{H}_{\mathrm{t}}=\mathrm{N}^{-1} \sum_{\mathrm{i}=1}^{\mathrm{N}}\left(\mathrm{h}_{\mathrm{it}}-1\right)^{2}$ e $\mathrm{h}_{\mathrm{it}}=\hat{\mathrm{w}}_{\mathrm{it}} / \mathrm{N}^{-1} \sum_{\mathrm{i}=1}^{\mathrm{N}} \hat{\mathrm{w}}_{\mathrm{it}} ; \mathrm{com}$ $\hat{\mathrm{x}}_{i, t}$ representando o PIBAGpc dos estados sem o componente de ciclos econômicos.

Novamente, conforme se observa a partir de (3), o teste $\log t$ não é aplicado diretamente nas séries dos PIBAGpc, mas, sim, nos coeficientes de transição relativos, $\mathrm{h}_{\mathrm{i}, \mathrm{t}}$. As séries do PIBAGpc têm alta variabilidade devido a questões como entressafras, clima, sazonalidades, oscilações de preços internacionais etc. Assim, para que os coeficientes de transição relativos estejam em conformidade com o teste - ou seja, para que os mesmos sejam capazes de captar a tendência de longo prazo das séries - seria necessário que as séries de PIBAGpc fossem superssuavizadas,

${ }^{14}$ Na prática, a variável PIBAGpc pode ser descrita como $\log y_{i, t}=b_{i, t} \cdot \mu_{t}+\kappa_{i, t}$ em que $\kappa_{i, t}$ representa um efeito de ciclo de negócios. Phillips e Sul sugerem que a remoção do componente de ciclos seja realizada por meio da utilização do filtro de Hodrick-Prescott (HP). Esta abordagem não requer nenhuma especificação a priori para $\mu_{\mathrm{t}}$ e é bastante cômoda, pois requer um único parâmetro de smooth como input.

15 Novamente, uma discussão em detalhes dessa metodologia pode ser vista em Penna e Linhares (2009). pois é de se esperar que a suavização através do filtro HP, conforme sugerem Phillips e Sul, não seja capaz de extrair completamente das séries do PIBAGpc a alta variabilidade gerada pelas questões levantadas acima.

Com efeito, a utilização de aproximações suaves para a formulação do painel (como, por exemplo, a extrapolação das séries com base em taxas de crescimento fixas entre períodos) pode contribuir no sentido de reduzir a variabilidade existente nas séries dos PIBAGpc estaduais. A aplicação do filtro HP a estas séries já suavizadas, por sua vez, atuaria no sentido de suavizar ainda mais as séries, o que traria propriedades desejáveis ao coeficiente de transição relativo $h_{i, t}$ para a aplicação do teste.

Em resumo, o teste log $t$ requer as tendências de longo prazo dos PIBAGpc estaduais e, devido a isto, é de se esperar que a formulação do painel através da extrapolação das séries com base em taxas de crescimento fixas entre períodos não seja capaz de alterar sobremaneira as estimativas aqui reportadas. Esta é a justificativa fundamental para que se possa trabalhar com o painel de dados descrito no Apêndice. ${ }^{16}$

Retornando à metodologia, sob hipótese nula, os coeficientes de (3) podem ser testados com base num teste $t$ unilateral, robusto à autocorrelação e heterocedasticidade. Para um nível de 5\%, por exemplo, a hipótese nula de convergência deve ser rejeitada se $t_{\hat{\beta}_{1}}<-1,65$. A rejeição da hipótese nula de convergência para todo o painel pode indicar a existência de pontos separados de equilíbrio ou múltiplos estados estacionários. Quando isso ocorre, pode-se ter a divergência de alguns membros do painel e/ou a formação de clubes de convergência. Neste contexto, Phillips e Sul (2007) propõem um algoritmo que aplica sequencialmente o teste $\log t$ e permite a identificação de clubes de convergência sem que se recorra às usuais características observáveis que condicionem o devido agrupamento deste

\footnotetext{
16 Ressalta-se que, apesar da adequação desta metodologia aos dados elaborados no apêndice, a manipulação dos mesmos através de outras metodologias pode gerar resultados inconsistentes.
} 
Tabela 2. O teste de Phillips e Sul (2007) para 1980-2008

$$
\log \frac{\mathrm{H}_{1}}{\mathrm{H}_{2}}-2 \log [\mathrm{L}(t)]=\beta_{0}+\beta_{2} \log t+\mathbf{u}_{\mathrm{t}}
$$

\begin{tabular}{ccccc}
\hline Grupo & Estados & Parâmetro & $\hat{\beta}$ & \\
\multirow{2}{*}{$\mathrm{G} 1$} & $\mathrm{AC}, \mathrm{GO}, \mathrm{MT}, \mathrm{MS}, \mathrm{PR}, \mathrm{RO}$, & $\beta_{0}$ & -5.117 & -6.529 \\
& $\mathrm{RR}, \mathrm{RS}, \mathrm{SC}, \mathrm{SP}$ e TO & $\beta_{1}$ & 0.735 & 2.759 \\
\multirow{2}{*}{$\mathrm{G} 2$} & \multirow{2}{*}{$\mathrm{ES}, \mathrm{MA}$ e MG } & $\beta_{0}$ & -2.875 & -12.975 \\
& \multirow{2}{*}{$\mathrm{G} 3$} & $\beta_{1}$ & 0.301 & 3.992 \\
& $\mathrm{AL}, \mathrm{AM}, \mathrm{AP}, \mathrm{BA}, \mathrm{CE}, \mathrm{DF}, \mathrm{PA}$, & $\beta_{0}$ & -2.085 & -5.838 \\
& $\mathrm{~PB}, \mathrm{PE}, \mathrm{PI}, \mathrm{RJ}, \mathrm{RN}$ e SE & $\beta_{1}$ & -0.133 & -1.098 \\
\hline
\end{tabular}

Fonte: Elaboração dos Autores.

clube (como, por exemplo, nível de escolaridade, estoque de capital, acesso ao crédito etc.).

No intuito de se averiguar a formação de clubes de convergência para os PIBAGpc estaduais, o coeficiente de transição relativa $\left(\mathrm{H}_{1} / \mathrm{H}_{\mathrm{t}}\right)$ é formulado e descartam-se os nove primeiros anos para o cômputo das estimativas. ${ }^{17}$ A aplicação do teste $\log t$ para todo o painel de dados dos PIBAGpc estaduais retorna uma estatística $t_{\hat{\beta}_{1}}=-10.42629$; como este valor é menor do que -1.65 , este resultado sugere que não há convergência global dos níveis dos PIBAGpc. Há, entretanto, espaço para a divergência de alguma unidade ou a possível formação de clubes de convergência. Ao se aplicar sequencialmente o teste $\log t$, a análise sugere que vêm se formando três clubes de convergência no Brasil. ${ }^{18}$ Os estados que compõem cada um dos clubes, as estatísticas de teste associadas à regressão do teste $\log t$ e um mapa com a distribuição regional dos mesmos são descritas na Tabela 2 .

Observa-se que o primeiro grupo é essencialmente formado pelas economias do Centro-Sul, com exceção dos estados do Acre,

17 Este descarte de 1/3 da amostra é sugerido por Phillips e Sul (2007), para que se elimine o efeito das observações iniciais no processo de convergência.

18 É importante ressaltar que este resultado é relativamente frágil em relação à amostra utilizada; por exemplo, ao se tomar o período 1985-2006 e descartando-se o Distrito Federal, o teste retorna a formação de apenas dois clubes de convergência (ES e MG são alocados no grupo de $P I B A G p c$ mais elevado e MA no de $P I B A G$ mais baixo).
Roraima e Rondônia. A estatística de teste para o grupo restante é de $t_{\hat{\beta}_{1}}=-25.189$; como este valor é menor do que 1.65, a possibilidade de formação de subgrupos de convergência permanece. $\mathrm{O}$ algoritmo identifica um segundo clube formado pelos estados do Espírito Santo, Maranhão e Minas Gerais, e um terceiro clube, formado basicamente pelos estados nordestinos (com exceção do Maranhão), Amazonas, Amapá e Pará, e pelo Distrito Federal e o Rio de Janeiro.

A trajetória de estado estacionário dos clubes pode ser aproximada pela média das séries filtradas, como se observa na Figura 3.

A Figura 3 elucida porque os testes usuais de $\sigma$ - convergência são violados para o caso brasileiro. A partir dele se observa que a trajetória de estado estacionário dos estados do terceiro grupo parece ter basicamente se estagnado; tal comportamento sugere que os estados pertencentes a este clube estariam muito próximos de seus PIBAGpc de estado estacionário. Já as trajetórias de estado estacionário do primeiro e do segundo clube, apesar de uma leve inflexão a partir da segunda metade dos anos 80, passam a crescer vigorosamente durante os anos 90 . Os diferenciais entre os PIBAGpc, condizente com a trajetória de estado estacionário de cada grupo, chamam a atenção: em 2008, a diferença entre os PIBAGpc de steady-state entre o primeiro e o segundo clube foi de $172 \%$, já o diferencial entre o primeiro clube e o terceiro foi de aproximadamente $490 \%$. 
Figura 3. Trajetórias de longo prazo dos clubes

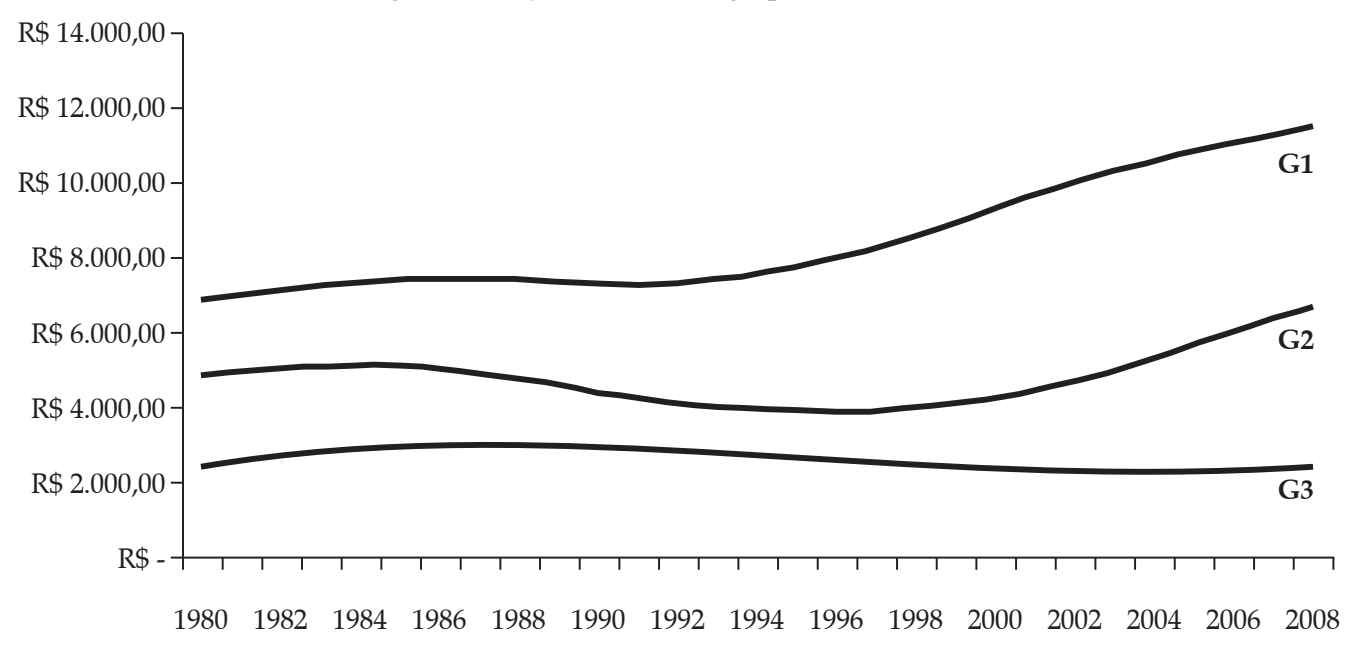

Fonte: Elaboração dos autores.

Como há convergência no longo prazo sempre que $h_{i, t} \rightarrow 1$, então se pode padronizar a trajetória de longo prazo para a unidade, de modo que seja possível analisar a dinâmica de transição de cada estado para sua respectiva posição de estado estacionário. As análises dos estados pertencentes ao primeiro, ao segundo e ao terceiro clube são feitas, respectivamente, nas Figuras 4, 5 e 6.

Figura 4. Dinâmica de transição do $1^{\mathrm{o}}$ clube (G1)

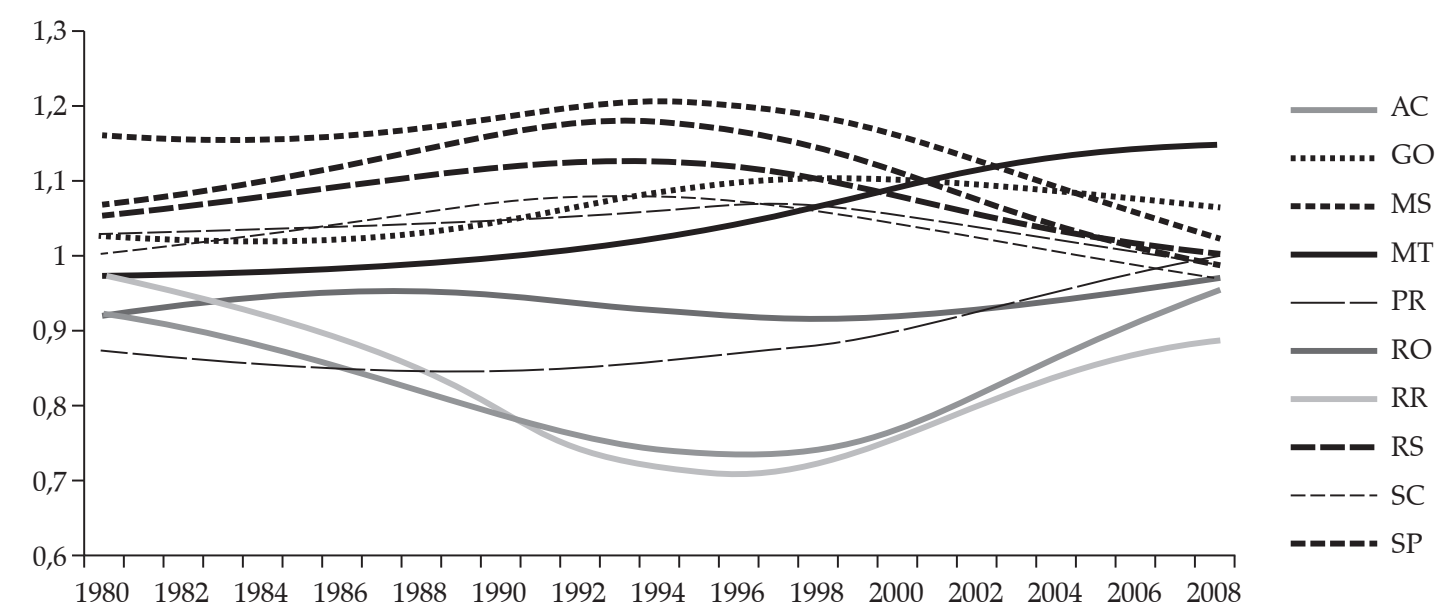

Fonte: Elaboração dos autores. 
Figura 5. Dinâmica de transição do $2^{\mathrm{O}}$ clube (G2)

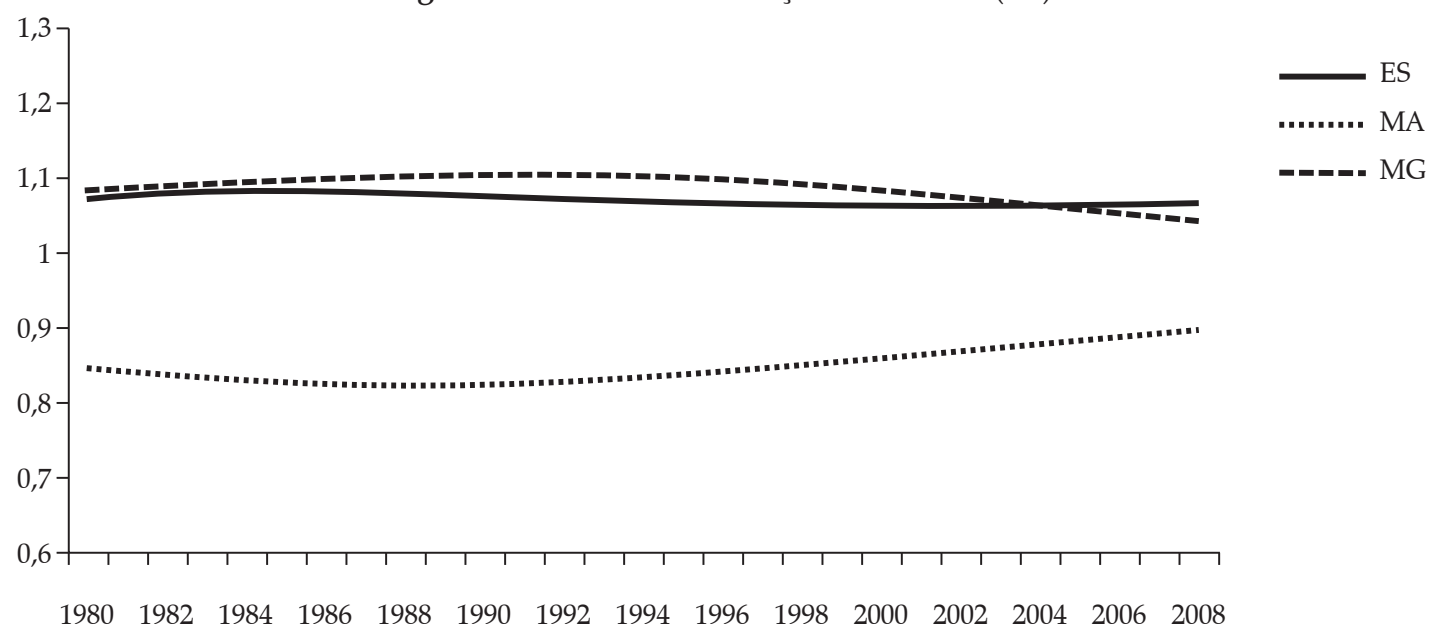

Fonte: Elaboração dos autores.

Figura 6. Dinâmica de transição do 3ํo clube (G3)

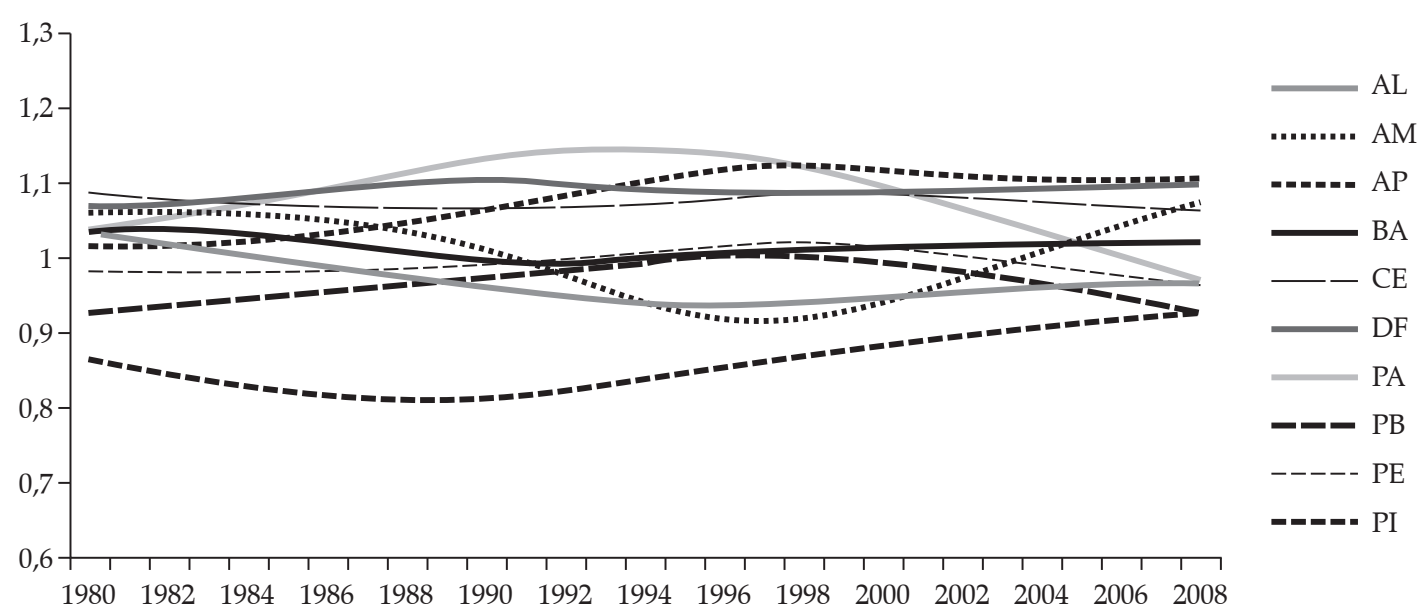

Fonte: Elaboração dos autores.

\section{Conclusões}

O presente trabalho trata do crescimento, do processo de convergência e da formação de clubes de convergência dos PIBs agropecuários per capita estaduais. Mais precisamente, o trabalho procura contribuir com a pesquisa inicial de Spohr e Freitas (2011), preenchendo algumas lacunas e seguindo o caminho sugerido pelos autores. $\mathrm{O}$ trabalho inicial sugere uma contradição entre os testes de $\beta$ - convergência e $\sigma$ - convergência. Esta contradição tem a vantagem de alavancar estudos na área e requer uma análise profunda do que pode motivar tais processos.

A análise aqui delineada reforça o trabalho de Spohr e Freitas (2011) de modo um pouco mais simples, embora mais ilustrativo no que se refere à violação da hipótese da $\sigma$ - convergência, e faz 
a constatação da $\beta$ - convergência de modo um pouco mais rigoroso.

O trabalho de Spohr e Freitas (2011) lida com dados em painel e faz uso de estimadores de mínimos quadrados ordinários com efeitos fixos para analisar a hipótese da $\beta$ - convergência; entretanto, a literatura empírica que trata do tema sugere que, em regressões como as estimadas pelos autores, há a ocorrência de correlação entre os regressores e o termo de erro, e isto possibilita diferentes tipos de vieses associados aos estimadores utilizados. A literatura sugere um aprimoramento com base num modelo dinâmico com dados em painel, em que se faz uso de estimadores que consideram o método generalizado dos momentos.

Aqui, foi conduzido o teste da $\beta$ - convergência com base no estimador GMM em primeiras diferenças, e de modo geral, os resultados encontrados no estudo-base continuam sendo evidenciados: a ocorrência da $\beta$ - convergência é sustentada, embora a $\sigma$ - convergência não.

Buscando dar uma resposta satisfatória a esta controvérsia, o trabalho adentra na questão da identificação de clubes de convergência. Para tanto, utiliza-se a metodologia proposta em Phillips e Sul (2007), a qual permite identificar clubes de convergência sob a hipótese de diversos tipos de heterogeneidade. Tal metodologia parece ser apropriada ao caso brasileiro devido à grande extensão territorial, às grandes diferenças climáticas e de solo, além de outras particularidades capazes de influenciar o desempenho agropecuário dos estados e regiões de maneira diferenciada.

A técnica proposta identifica três clubes de convergência: o primeiro tem elevado PIB agropecuário per capita de steady-state, $\mathrm{R} \$$ $11.451,51$, e é formado por Acre, Goiás, Mato Grosso $^{19}$ e Mato Grosso do Sul, Paraná, Roraima,

19 O caso de Mato Grosso parece chamar a atenção: como se pode observar na Figura 4, se esta unidade permanecer crescendo no mesmo ritmo que cresceu na década de 90, é possível que tal economia seja diferente de seu grupo (séries temporais mais longas possivelmente retornariam este resultado).
Rondônia, Rio Grande do Sul, Santa Catarina e São Paulo; o segundo tem algo próximo de R\$ 6.665,64 e é formado por Espírito Santo, Maranhão e Minas Gerais; o terceiro clube é formado pelos demais estados e tem PIBAGpc muito aquém dos outros, R\$2.339,03. ${ }^{20}$

Uma análise das trajetórias de longo prazo dos clubes reforça a ideia de que os testes de $\sigma$ convergência, de fato, tenderiam ser violados para o caso brasileiro. Também se observa que os PIBAGpc dos estados do terceiro grupo parece ter estancado, o que sugere que estes estados devem estar muito próximos de seus PIBAGpc de estado estacionário. As trajetórias do primeiro e do segundo grupo, entretanto, permanecem a crescer. Embora haja uma elevada segregação entre clubes, é possível se constatar $\sigma$-convergência "intraclubes".

Com efeito, e posto que Barro e Sala-i-Martin (2004) sustentam que $\sigma$-convergência implica necessariamente $\beta$-convergência, a identificação destes clubes de convergência parece sugerir que os estados pertencentes a cada um destes clubes estejam, de fato, experimentando $\beta$-convergência (condicional). Isto é evidenciado pela Figura 2, que sugere uma possível não linearidade para os grupos de estados identificados, ou seja, diferentes retas de regressão para cada um destes grupos. ${ }^{21}$

De modo geral, o trabalho procurou levantar pontos e traçar diretrizes para os estudos e políticas que ainda virão. Discutir políticas públicas associadas ao processo de formação de clubes, principalmente quando este processo é visto em âmbito rural, é algo complexo e que transpassa o escopo desta nota, entretanto, a análise aqui delineada põe em voga a necessidade de esforços para que se investigue o que tem motivado este processo e de que maneira pode ser possível tomar proveito ou buscar se desvencilhar do mesmo.

\footnotetext{
20 Dados para 2008, em R\$ de 2010.

21 Poderia se pensar em estimar o processo de convergência associando variáveis dummy às técnicas de painel dinâmico abordadas na Seção 3; entretanto, tais técnicas requerem que o modelo estimado seja linear (ROODMAN, 2009). Avanços nesta área ficam como propostas de trabalhos futuros, vide nota de rodapé \#11.
} 


\section{Referências bibliográficas}

ALMEIDA, E. S., PEROBELLI, F. S. e FERREIRA, P. G. C. Existe convergência espacial da produtividade agrícola no Brasil? Revista de Economia e Sociologia Rural, v. 46, n. 01, p. 31-52, jan/mar 2008.

BARRETO, R. C. S. e ALMEIDA, E. A contribuição da pesquisa para convergência e crescimento da renda agropecuária no Brasil. Rev. Econ. Sociol. Rural, Brasília, v. 47, n. 3, Sept. 2009.

BARRO e SALA-I-MARTIN. Economic Growth. New York: McGraw-Hill, 2004.

BOND, S., HOEFFLER, A. e TEMPLE, J. GMM Estimation of Empirical Growth Models. University of Oxford, Institute for Fiscal Studies, 2001.

CASELLI, F., ESQUIVEL, G. e LEFORT, F. Reopening the convergence debate: a new look at cross-country growth empirics. Journal of Economic Growth, v. 1, n. 3, p. 363-389, 1996.

GREENE, W. Econometric Analysis. 5a ed. New York: Prentice Hall, 2003.

ISLAM, N. Growth empirics: a panel data approach. Quarterly Journal of Economics, v. 110, n. 4, p. 1127-1170, 1995.

OLIVEIRA JUNIOR, J. N., CASTELAR, I. e FERREIRA, R. T. Convergência Microrregional no Setor Agrícola usando um Modelo Threshold. Revista ANPEC, v. 10, p. 553-576, 2009.

PENNA, C. M. e LINHARES, F. Convergência e Formação de Clubes no Brasil sob a Hipótese de Heterogeneidade no Desenvolvimento Tecnológico. Revista Econômica do Nordeste, v. 40, p. 781-796, 2009.
PENNA, C. M e LINHARES, F. Teste da Convergência do PIB per Capita da Agropecuária no Brasil: Comentários e Contribuições, 2011. (working paper apresentado no Encontro Regional de Economia do Nordeste BNB/ ANPEC 2011)

PHILLIPS, P. C. B. e SUL, D. Transition modeling and econometric convergence tests. Econometrica, Econometric Society, v. 75, n. 6, p. 1771-1855, nov. 2007.

QUAH, D. Twin peaks: growth and convergence in models of distribution dynamics. Economic Journal, v. 106, p. 1045-1055, 1996.

MAASOUMI, E., RACINE, J. e STENGOS, T. Growth and convergence: a profile of distribution dynamics and mobility. Journal of Econometrics, v. 136, n. 2, p. 483-508, 2007.

ROODMAN, D. How to do xtabond2: An introduction to difference and system GMM in Stata. Stata Journal, StataCorp LP, v. 9, n. 1, p. 86-136, mar. 2009.

SHIN, Y. Bootrstrap-based Bias Corrected Within Estimation of Threshold Regression Models in Dynamic Panels, 2008. (mimeo)

SILVA, A. L. F. E. e OLIVEIRA JUNIOR, J. N. Convergência Condicional ou Clubes? Uma Aplicação da Econometria Espacial para o Setor Agrícola na Amazônia Legal - 1996-2006. In: XXXVIII Econtro Nacional de Economia - ANPEC, 2010, Salvador. Anais do XXXVIII Econtro Nacional de Economia - ANPEC, 2010.

SPOHR, G. e FREITAS, A. F. Teste da Convergência do PIB Per capita da Agropecuária no Brasil entre 1980 e 2004. Revista de Economia e Sociologia Rural, Piracicaba, SP, v. 49, n. 02, p. 341-368, abr/jun 2011. 


\section{Apêndice - formulação dos dados}

A base de dados para a formulação dos PIBAGpc estaduais foi feita com base em duas séries: (i) a do PIB estadual da agropecuária, disponível em R\$ de 2000 para o ano de 1980 e para todos os anos do período 1985-2008. Entre 1980-1985, esta série foi extrapolada supondo-se uma taxa de crescimento constante entre os períodos. Para o caso do Tocantins, a extrapolação levou em conta 1980-1988. Também são necessárias as séries (ii) da população residente rural, que é dada em número de habitantes. Os dados disponíveis para esta segunda variável são os anos de 1980, 1991, 1996, 2000 e 2007. Para 2007 não se tem informação para o Distrito Federal. Como se está abordando o período de 1985 a 2006, foi necessário extrapolar esta variável dentre os anos supracitados supondo uma taxa de crescimento populacional rural constante entre os períodos. Para o caso do Distrito Federal, o período 2000-2007 foi preenchido supondo-se que a taxa de crescimento populacional foi a mesma para o período 1996-2000.

Dividindo-se os dados do PIB da agropecuária pela população rural, obtém-se o PIB per capita do setor agropecuário. Fazendo-se uso do deflator implícito do PIB da agropecuária é possível converter estes dados para R\$ de 2010. As fontes e as séries construídas e utilizadas estão disponíveis em www.ler.ufc.br/ pib_agropecuaria.xlsx.

As séries aqui apresentadas diferem das de Spohr e Freitas (2011) em vários aspectos. O primeiro deles é o de que o PIBAGpc de Mato Grosso do Sul não é o mais elevado em 2004, como frisam os autores. No referido ano, o estado com PIBAGpc mais elevado foi Mato Grosso, com aproximadamente R\$32.000,00, seguido por Goiás, com R\$19.012,69, e só em seguida desponta o estado de Mato Grosso do Sul, com R\$16.376,60 (novamente, todos estes valores estão em $\mathrm{R} \$$ de 2010). ${ }^{22}$

${ }^{22}$ Ao se fazer uma relativização entre os PIBs agropecuários per capita dos estados para os anos de 1980 e 2008, tomando-se em 1980 e em 2008 o estado com maior PIBAGpc como referência, também se observa que o estado de Mato Grosso do Sul perde a liderança para o estado de Mato Grosso entre 1980 e 2008, e que estados como São Paulo e Rio Grande do Sul cedem lugar aos estados de Mato Grosso do Sul e Goiás. Fenômenos de catch-up e de pushing back como estes chamaram a atenção de Quah (1996), que se propôs a estudar o processo de convergência através da análise do comportamento dinâmico da intradistribuição de renda, assim como de Maasoumi, Racine e Stengos (2007), que investigaram o processo de formação de clubes de convergência com base em modelos não paramétricos que lidam com o conceito de entropia. A aplicação destas metodologias ainda não foi aplicada aos PIBAGpc e pode ser tema para trabalhos futuros convenientes. 
Algumas críticas podem ser feitas em termos de confiabilidade dos dados, principalmente ao se argumentar que os mesmos podem impor algum tipo de viés à análise. Esta nota se concentra principalmente na contradição dos resultados da $\beta$ e $\sigma$-convergência, assim como na constatação de clubes de convergência; assim, o foco aqui não é se obter estimativas precisas do coeficiente $\beta$, embora nossos resultados com base no painel estejam bem próximos do reportado no trabalho de Spohr e Freitas (2011). 
